\title{
Effect of $n-3$ polyunsaturated fatty acids on ischemic heart disease and cardiometabolic risk factors: a two-sample Mendelian randomization study
}

\author{
Bayi $X u^{1}$, Zhixia $X^{2}$, Duanmin $X u^{1}$ and $X u e r u i \operatorname{Tan}^{3^{*}}(\mathbb{0}$
}

\begin{abstract}
Background: The cardioprotective ability of n-3 polyunsaturated fatty acids (PUFAs) is controversial. Most studies suggest a specific role for PUFAs in cardioprotection from ischemic heart disease (IHD). However, few studies have used genetic biomarkers of n-3 PUFAs to examine their potential relationships with IHD. This study aimed to use Mendelian randomization to evaluate whether genetically-predicted n-3 PUFAs affect IHD and cardiometabolic risk factors (CRFs).
\end{abstract}

Methods: Genetic variants strongly $\left(p<5 \times 10^{-8}\right)$ and independently $\left(r^{2}>0.1\right)$ associated with $n-3$ PUFAs were derived from the CHARGE Consortium (including 8,866 subjects of European ancestry) and were used as instrumental variables (IVs) for evaluating the effect of n-3 PUFAs, including a-linolenic acid (ALA), docosapentaenoic acid (DPA), docosahexaenoic acid (DHA), and eicosapentaenoic acid (EPA). Data on the associations between the IVs and IHD, myocardial infarction, and CRFs (including diabetes, lipids, blood pressure, body mass index, and waist-to-hip ratio (WHR)) were obtained from the UK Biobank SOFT CAD GWAS with the CARDloGRAMplusC4D 1000 Genomes-based GWAS (113,937 IHD cases and 339,115 controls), the Myocardial Infarction Genetics and CARDloGRAM Exome consortia (42,335 MI cases and 78,240 controls), the DIAbetes Genetics Replication And Meta-analysis consortium (26,676 diabetes mellitus cases and 132,532 controls), the Global Lipids Genetics Consortium ( $n=196,475)$, the International Consortium for Blood Pressure $(n=69,395)$, and the meta-analysis of GWAS for body fat distribution in the UK Biobank and Genetic Investigation of Anthropometric Traits $(n=694,649)$.

Results: Genetically-predicted higher ALA was associated with lower risk of IHD, type 2 diabetes (T2D), and lower serum lipids. The effect size per 0.05-unit increase (about 1 standard deviation) in plasma ALA level) was - 1.173 (95\% confidence interval -2.214 to -0.133 ) for IHD. DPA and EPA had no association with IHD but were associated with a higher risk of T2D, higher levels of lipids or WHR. DHA had no association with IHD or CRFs.

Conclusions: Our study suggests a benefit of ALA for IHD and its main risk factors. DHA, DPA, and EPA had no association with IHD but were partly associated with increasing cardiometabolic risk factors.

Keywords: N-3 polyunsaturated fatty acids, Mendelian randomization, Ischemic heart disease, Cardiometabolic risk factors

\footnotetext{
*Correspondence: xueruitan@sina.com

${ }^{3}$ Clinical Research Center, First Affiliated Hospital of Shantou University

Medical College, Shantou 515041, Guangdong, China

Full list of author information is available at the end of the article
}

(c) The Author(s) 2021. Open Access This article is licensed under a Creative Commons Attribution 4.0 International License, which permits use, sharing, adaptation, distribution and reproduction in any medium or format, as long as you give appropriate credit to the original author(s) and the source, provide a link to the Creative Commons licence, and indicate if changes were made. The images or other third party material in this article are included in the article's Creative Commons licence, unless indicated otherwise in a credit line to the material. If material is not included in the article's Creative Commons licence and your intended use is not permitted by statutory regulation or exceeds the permitted use, you will need to obtain permission directly from the copyright holder. To view a copy of this licence, visit http://creativecommons.org/licenses/by/4.0/. The Creative Commons Public Domain Dedication waiver (http://creativecommons.org/publicdomain/zero/1.0/) applies to the data made available in this article, unless otherwise stated in a credit line to the data. 


\section{Background}

N-3 polyunsaturated fatty acids (PUFAs) include plantderived $\alpha$-linolenic acid (ALA) and marine-derived docosahexaenoic acid (DHA), docosapentaenoic acid (DPA), and eicosapentaenoic acid (EPA). DHA, DPA, and EPA are long-chain n-3 fatty acids. Evidence from observational, experimental studies, and randomized controlled trials (RCT) show that n-3 PUFAs from diet or supplements confer protection against cardiovascular disease (CVD) and relevant risk factors, including cardiac death, ischemic heart disease (IHD), ischemic stroke, heart failure, and blood pressure [1, 2]. However, integrated analyses of these studies have found null, little, or inconsistent results, no matter whether in primary or in secondary prevention of CVD [3-5]. Recent evidence from high-quality large RCTs also suggests that n-3 PUFA intake probably makes little or no difference for coronary heart mortality or events [6-9]. The nutrition recommendations for n-3 PUFA supplements or seafood for cardiovascular benefits have been debated in recent years, but without consensus being reached [10]. These controversies may be confounded by background dietary consumption of fish, health status, medical treatment of IHD, socioeconomic position, lifestyle, different study populations, and different definitions of CVD and study endpoints $[1,10]$.

Mendelian randomization (MR) studies use germline genetic variants as intermediate instrumental variables (IVs) to assess causal relationships in a non-experimental setting. As genetic variants are determined at conception, MR studies are less susceptible to confounders than observational studies and are not affected by disease status, thereby avoiding reverse causation bias. MR studies can be regarded as "natural" RCTs and have been applied to examine genetic predisposition conferred by several genes on IHD [11]. In the present study, we conducted a two-sample MR study to assess the effect of genetically predicted n-3 PUFAs on IHD, using genetically instrumented n-3 PUFAs from previous studies and a very large case-control dataset of IHD from public consortia. In addition, cardiometabolic risk factors (CRFs) of IHD, including type 2 diabetes (T2D), hyperlipidemia, hypertension, and abdominal obesity were similarly assessed.

\section{Methods}

\section{Genetic instruments for $\mathrm{n}-3$ PUFAs}

Genetic instruments for n-3 PUFAs were obtained from published genome-wide association studies (GWAS) conducted by the Cohorts for Heart and Aging Research in Genomic Epidemiology (CHARGE) consortium [12]. The CHARGE Consortium is a design of prospective meta-analyses of GWAS from five population-based cohorts comprising 8,866 subjects of European ancestry and can be used for proxy plasma levels of $n-3$ fatty acids. The different $\mathrm{n}-3$ PUFAs share a common metabolic pathway and single nucleotide polymorphisms (SNPs) known to influence one n-3 PUFA typically also have strong effects on the others [12]. All SNPs chosen as IVs were associated with the relevant n-3 PUFAs (ALA, DHA, DPA, and EPA) and reached genome-wide significance $\left(p<5 \times 10^{-8}\right)$ (Additional file 1: Tables S1-S4). The linkage disequilibrium (LD) between instrumental SNPs was obtained using LDlink [13], a web-based LD analysis tool designed to easily query pair-wise LD between SNPs in specific population groups.

Genetic associations with IHD, T2D, lipids, blood pressure, body mass index (BMI), and waist-to-hip ratio (WHR)

Genetic associations with IHD were obtained from the published meta-analysis of UK Biobank SOFT CAD GWAS with the CARDIoGRAMplusC4D 1000 Genomes-based GWAS and the Myocardial Infarction Genetics and CARDIoGRAM Exome, which is the most up-to-date GWAS of IHD applied to the UK Biobank and involved 113,937 IHD cases and 339,115 controls in total. The UK Biobank SOFT CAD GWAS comprised 10,801 cases and 137,371 controls, and $94 \%$ of the participants were of self-reported European ancestry [14]. SOFT CAD phenotypes in UK Biobank encompassed individuals with fatal or nonfatal myocardial infarction (MI), percutaneous transluminal coronary angioplasty (PTCA), or coronary artery bypass grafting (CABG), chronic IHD, and angina. Controls were those who were free from case status. The CARDIoGRAMplusC4D 1000 Genomes-based GWAS consortium comprised 60,801 cases and 123,504 controls and most of the participants (77\%) were of European descent [15]. IHD status was determined from clinical diagnosis, medical records and self-reports of medication usage, procedures such as revascularization, and other evidence of stenosis such as from coronary angiography. The Myocardial Infarction Genetics and CARDIoGRAM Exome consortium comprised 42,335 cases and 78,240 controls and all of the participants were of European descent [16].

Genetic associations with diabetes were obtained from the DIAbetes Genetics Replication and Meta-analysis (DIAGRAM) consortium. The DIAGRAM consortium is a grouping of researchers with shared interests in performing large-scale studies to characterize the genetic basis of T2D. The stage 1 analyses comprised a total of 26,676 T2D cases and 132,532 control participants of European descent [17]. Genetic associations with lipids, including high-density lipoprotein (HDL) cholesterol, low-density lipoprotein (LDL) cholesterol, total cholesterol (TC), and triglycerides (TG), were obtained from the Global Lipids Genetics Consortium (GLGC), which 
included 188,577 European-ancestry individuals and 7,898 non-European ancestry individuals [18]. Genetic associations with blood pressure, systolic and diastolic blood pressure (SBP and DBP), were obtained from the International Consortium for Blood Pressure (ICBP), which included 69,395 individuals of European ancestry [19]. Genetic associations with BMI and WHR were obtained from the meta-analysis of GWAS for body fat distribution in UK Biobank and Genetic Investigation of Anthropometric Traits (GIANT) and included 694,649 individuals of European ancestry [20].

\section{Statistical analysis}

In this study, we conducted a two-sample MR analysis using R version 4.0.3 (R Foundation for Statistical Computing, Vienna, Austria) and the R package for Mendelian randomization (version 0.5.1). The main methods included inverse-variance weighted (IVW), Mendelian randomization-Egger (MR-Egger), MR-PRESSO (Mendelian Randomization Pleiotropy Residual Sum and Outlier), and weighted median (WM) methods [21-23]. These methods have their advantages and can complement each other, providing a more reliable causal inference for our study. The MR-PRESSO method can identify and exclude SNPs that most likely display pleiotropic effects [21]. All statistical tests were two-sided, and $p<0.05$ was considered statistically significant.

As shown in Fig. 1, MR analysis relies on three stringent assumptions [24]: (1) the genetic instruments (SNPs) used as IVs are strongly predictive of n-3 PUFA, (2) the association of genetic instruments with IHD is not confounded by measured or unmeasured factors, (3) the effect of the genetic instrument on IHD should be fully mediated via n-3 PUFAs and not through any alternative causal pathways. Pleiotropic genetic instruments violate the MR assumptions and may lead to false-positive results, interfering causal inference. We identified potential pleiotropic genetic instruments in the following ways. First, we examined the latest representative GWAS results (the DIAGRAM consortium, the GLGC consortium, the ICBP consortium, the UK Biobank, and the GIANT) to identify associations between our genetic instruments and potential biological confounding factors, including diabetes, lipids, blood pressure, BMI, and WHR. Secondly, potential pleiotropic effects of the genetic instruments were investigated by searching the

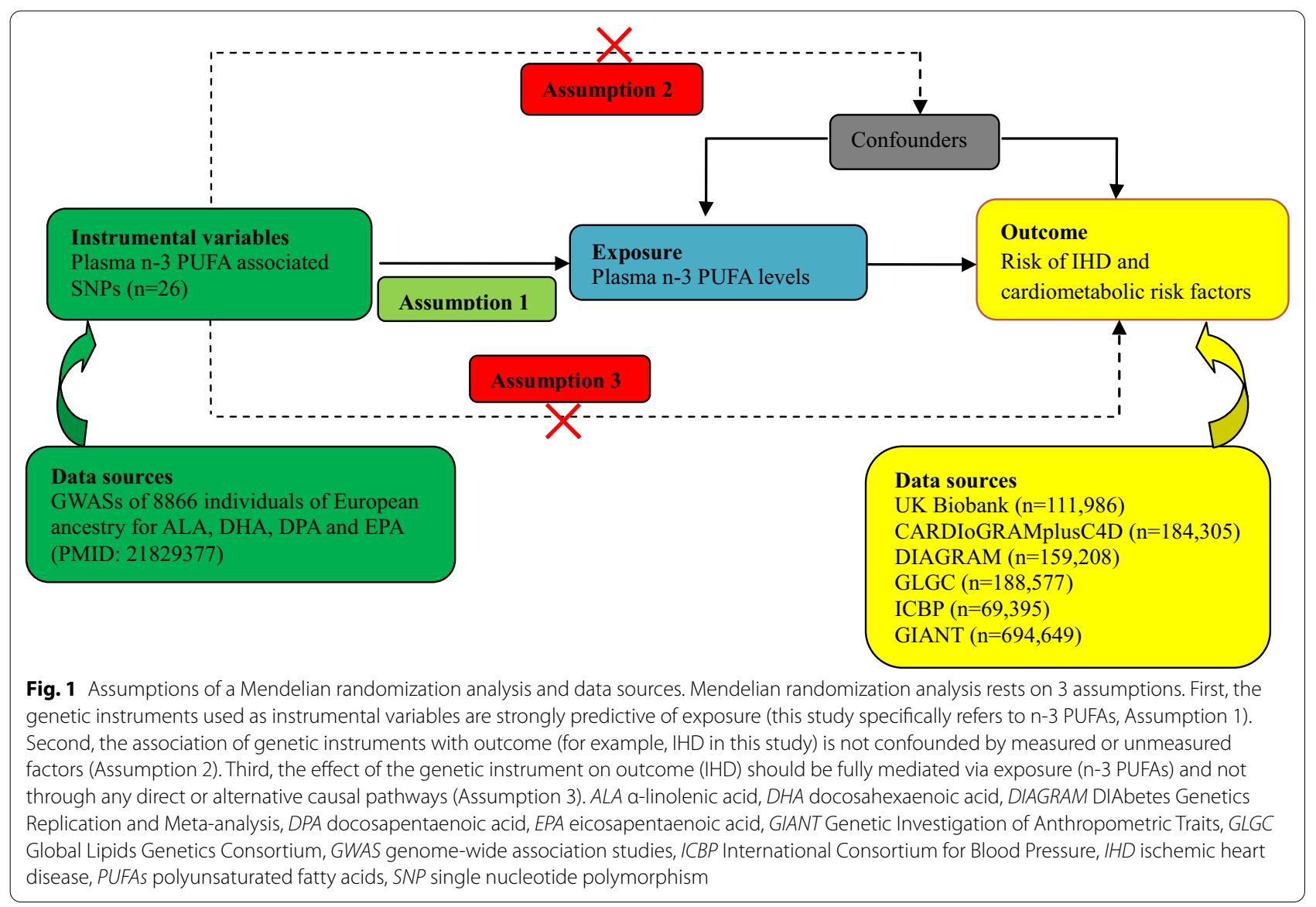


literature. Genetic instruments with potential pleiotropy were excluded.

\section{Results}

\section{Genetic instruments for $n-3$ PUFAs}

After removing SNPs in linkage disequilibrium with the other SNPs $\left(r^{2}>0.1\right), 31$ genome-wide significant $\left(p<5 \times 10^{-8}\right)$ n-3 PUFA-associated SNPs were retained for subsequent analysis (Additional file 1: Table S5). According to these 31 instrumental SNPs, genetic associations with IHD and CRFs were found from the corresponding GWAS database (Additional file 1: Tables S6-S16). Then, we examined the GWAS database to identify potential pleiotropic SNPs and found SNPs that were associated with potential confounders. Three SNPs were associated with HDL (rs174547, rs7942717, and rs174538, Additional file 1: Table S10), three SNPs were associated with TC (rs174547, rs780094, and rs174538,
Additional file 1: Table S11), four SNPs were associated with TG (rs174547, rs7942717, rs780094, and rs174538, Additional file 1: Table S12), and one SNP was associated with BMI (rs780094, Additional file 1: Table S16). As pleiotropic SNPs are likely to violate the assumptions underlying MR, these SNPs were excluded from the set of IVs. No additional pleiotropic SNPs were further identified by other methods. Finally, 26 n-3 PUFA-associated SNPs were retained for IVs. These SNPs explained 0.940\% $(0.148-3.502 \%)$ of the variance in plasma $n-3$ PUFA levels (Table 1).

The strength of the genetic instruments was evaluated by the F-statistic. According to methods of a previous study $[25,26]$, summary statistics from a previous $n-3$ PUFA GWAS [12, 27] were used to calculate the F-statistic. Our results showed the F-statistic of all instrumental SNPs was $>10$ (mean 85, from 13 to 322 ) (Table 1), indicating strong genetic instruments for n-3 PUFAs [28].

Table 1 Characteristics of the SNPs associated with plasma levels of $n-3$ polyunsaturated fatty acids (PUFAs)

\begin{tabular}{|c|c|c|c|c|c|c|c|c|c|c|}
\hline n-3 PUFA & Nearest gene & SNP & Chr. position & EA/NEA & EAF & Effect & SE & $p$-value & VE (\%) & $F$ \\
\hline \multirow[t]{4}{*}{ ALA } & FEN1 & rs412334 & 11:61316837 & $t / c$ & 0.16 & -0.0118 & 0.0016 & $9.72 \mathrm{E}-14$ & 0.342 & 30 \\
\hline & C11orf9 & rs198464 & $11: 61278197$ & $a / g$ & 0.50 & -0.0057 & 0.0009 & $2.48 E-11$ & 0.148 & 13 \\
\hline & C11orf10 & rs740006 & $11: 61314444$ & $t / c$ & 0.90 & 0.0163 & 0.0025 & $1.32 \mathrm{E}-10$ & 0.440 & 39 \\
\hline & C11orf9 & rs17762402 & 11:61309777 & $a / g$ & 0.07 & -0.0186 & 0.0029 & $1.56 \mathrm{E}-10$ & 0.411 & 37 \\
\hline $\mathrm{DHA}$ & ELOVL2 & rs 2236212 & 6:10995015 & $c / g$ & 0.41 & -0.1132 & 0.0141 & $1.26 \mathrm{E}-15$ & 0.646 & 58 \\
\hline \multirow[t]{15}{*}{ DPA } & ELOVL2 & rs3734398 & 6:10982973 & $t / c$ & 0.57 & -0.0404 & 0.0029 & $9.61 E-44$ & 2.667 & 243 \\
\hline & FEN1 & rs412334 & $11: 61560261$ & $t / c$ & 0.16 & 0.0554 & 0.0052 & $1.40 \mathrm{E}-26$ & 2.750 & 251 \\
\hline & C11orf10 & rs740006 & 11:61557868 & $t / c$ & 0.9 & -0.0764 & 0.0079 & $4.50 E-22$ & 3.502 & 322 \\
\hline & FADS3 & rs7394871 & $11: 61652514$ & $a / c$ & 0.05 & -0.0637 & 0.0078 & $3.56 E-16$ & 1.285 & 115 \\
\hline & FADS2 & rs498793 & 11:61624705 & $t / c$ & 0.43 & 0.0307 & 0.0038 & $5.84 \mathrm{E}-16$ & 1.540 & 139 \\
\hline & SYCP2L & rs12199131 & 6:10932569 & $a / g$ & 0.26 & 0.0267 & 0.0033 & $7.69 E-16$ & 0.914 & 82 \\
\hline & RAB3IL1 & rs174472 & 11:61671956 & $a / g$ & 0.58 & 0.0274 & 0.0037 & $5.74 \mathrm{E}-14$ & 1.219 & 109 \\
\hline & C11orf9 & rs17762402 & 11:61553201 & $\mathrm{a} / \mathrm{g}$ & 0.06 & 0.0805 & 0.0109 & $1.42 \mathrm{E}-13$ & 2.437 & 221 \\
\hline & SYCP2L & rs6928281 & 6:10908917 & $\mathrm{t} / \mathrm{g}$ & 0.72 & 0.0226 & 0.0032 & $8.04 E-13$ & 0.686 & 61 \\
\hline & C11orf9 & rs198464 & $11: 61521621$ & $a / g$ & 0.49 & 0.0191 & 0.0028 & $7.47 E-12$ & 0.608 & 54 \\
\hline & FADS2 & rs17156442 & $11: 61614023$ & $t / c$ & 0.05 & -0.0513 & 0.0077 & $2.09 E-11$ & 0.833 & 74 \\
\hline & BEST1 & rs1109748 & $11: 61722645$ & $a / c$ & 0.07 & -0.0400 & 0.0068 & $5.09 E-09$ & 0.694 & 62 \\
\hline & ELOVL2 & rs6936315 & 6:11035972 & $t / c$ & 0.84 & 0.0244 & 0.0043 & $1.34 \mathrm{E}-08$ & 0.533 & 48 \\
\hline & FTH1 & rs10792320 & 11:61746291 & $a / c$ & 0.65 & 0.0160 & 0.0030 & $8.49 E-08$ & 0.388 & 35 \\
\hline & BEST1 & rs2727266 & 11:61704334 & $\mathrm{a} / \mathrm{g}$ & 0.93 & 0.0308 & 0.0058 & $8.86 \mathrm{E}-08$ & 0.412 & 37 \\
\hline \multirow[t]{6}{*}{ EPA } & FADS3 & rs7394871 & $11: 61652514$ & $a / c$ & 0.05 & -0.0912 & 0.0128 & $1.13 E-12$ & 0.494 & 44 \\
\hline & ELOVL2 & rs3798713 & $6: 11008622$ & $c / g$ & 0.42 & 0.0350 & 0.0050 & $1.93 E-12$ & 0.373 & 33 \\
\hline & BEST1 & rs1109748 & $11: 61722645$ & $a / c$ & 0.07 & -0.0535 & 0.0092 & $5.46 \mathrm{E}-09$ & 0.233 & 21 \\
\hline & FEN1 & rs412334 & 11:61560261 & $\mathrm{t} / \mathrm{c}$ & 0.16 & 0.0440 & 0.0081 & $4.59 \mathrm{E}-08$ & 0.325 & 29 \\
\hline & FADS2 & rs498793 & 11:61624705 & $t / c$ & 0.43 & 0.0351 & 0.0064 & $5.11 \mathrm{E}-08$ & 0.377 & 34 \\
\hline & MAT2B & rs1145652 & 5:164764087 & $a / g$ & 0.87 & 0.0356 & 0.0066 & $8.39 E-08$ & 0.179 & 16 \\
\hline
\end{tabular}

ALA a-linolenic acid, Chr. chromosome, DHA docosahexaenoic acid, DPA docosapentaenoic acid, EA effect allele, EAF effect allele frequency, EPA eicosapentaenoic acid, F F-statistic, NEA non-effect allele, PUFAs polyunsaturated fatty acids, SE standard error, SNP single nucleotide polymorphism, VE variation explained

$\operatorname{VE}(\%)=\left(2 \times\right.$ Effect $^{2} \times$ EAF $\times(1-$ EAF)/var $(n-3$ PUFAs $)) \times 100, \operatorname{var}(n-3$ PUFA) is the variance of $n-3$ PUFA and was deduced based on literature data (PMID: 27490808$)$. F-statistic is a measure of the strength of the genetic instrument and is calculated as follows: $F=\left(R^{2} \times(n-1-k)\right) /\left(\left(1-R^{2}\right) \times k\right)$, where $R^{2}=V E(\%), n=s a m p l e ~ s i z e$, $\mathrm{k}=$ number of instrumental variables 


\section{Associations of $n-3$ PUFAs with IHD}

Figure 2 and Table S17 (Additional file 1) showed higher ALA was associated with lower IHD risk, based on IVW $(p=0.027)$ and WM $(p=0.006)$, but with inconsistent results from MR-Egger $(p>0.05)$ and MR-PRESSO $(p>0.05)$. ALA was not associated with MI, with consistent results, based on all 4 methods (IVW, WM, MREgger, and MR-PRESSO) (all $p>0.05$ ). DPA and EPA were not significantly associated with IHD or MI, with consistent results from all 4 methods (all $p>0.05$ ). MR-Egger intercepts $p>0.05$ suggested little evidence of directional pleiotropy in all analyses. As WM, MR-Egger and MRPRESSO methods require a minimum of 3 IVs, the associations of DHA with IHD, MI and CRFs were estimated by IVW only, and the results showed that DHA was not associated with IHD or MI (all $p>0.05$ ).

\section{Associations of n-3 PUFAs with T2D, lipids, blood pressure, BMI, and WHR}

We also tested the association of n-3 PUFAs with CRFs. As shown in Fig. 2 and Table S17 (Additional file 1), ALA was associated with a lower risk of T2D (IVW, MRPRESSO) and lower HDL (IVW, WM, MR-PRESSO), LDL (IVW, WM), TG (IVW, WM), and TC (IVW, WM) levels $(p<0.05$ or 0.001$)$. ALA had no association with SBP, DBP, BMI, or WHR (all $p>0.05$ ). There was little evidence of directional pleiotropy based on the MR-Egger intercept (all $p>0.05$ ).

DPA was associated with a higher risk of T2D (IVW, WM, MR-PRESSO) and higher HDL (IVW), LDL (IVW, WM, MR-PRESSO), TC (IVW, WM, MR-PRESSO), and WHR (IVW, WM, MR-PRESSO) $(p<0.05$ or 0.001$)$. DPA had no association with TG, SBP, DBP, or BMI (all $p>0.05$ ), and there was little evidence of directional pleiotropy based on the MR-Egger intercept (all $p>0.05$ ). EPA was associated with higher LDL (IVW, WM), TC (IVW, WM), WHR (IVW), and lower SBP (MR-Egger), DBP (MR-Egger). As for TG, there were inconsistent results: WM indicated TG was a risk factor whereas MR-Egger indicated TG was a protective factor (both $p<0.05)$. EPA had no association with T2D, HDL, or BMI (all $p>0.05$ ). Based on the MR-Egger intercept, there was potential pleiotropy between EPA and LDL $(p=0.021)$, TC $(p=0.044)$, TG $(p=0.003), \operatorname{SBP}(p=0.012)$, and DBP $(p<0.001)$. In this case, the MR-PRESSO results should prevail and we conclude that EPA had no association with LDL, TC, TG, SBP, or DBP (all $p>0.05$ ). DHA had no association with CRFs.

\section{Discussion}

This MR study showed that a genetic predisposition toward higher plasma ALA level is associated with a lower risk of IHD, but not MI. The effect size (beta coefficient) per 0.05-unit increase (about $1 \mathrm{SD}$ ) in plasma ALA level was -1.173 (95\% confidence interval -2.214 to -0.133 ) for IHD. In contrast, genetically-predicted levels of marine-derived n-3 PUFAs (DHA, DPA, and EPA) had no association with IHD or MI.

On primary prevention of CVD, Abdelhamid et al. [3] found increased ALA may slightly reduce the risk of cardiovascular events, coronary heart disease (CHD) mortality, and arrhythmia, and Pan et al. [29] found dietary ALA is associated with a moderately lower risk of fatal CHD, with each $1 \mathrm{~g} / \mathrm{d}$ increment of ALA intake being associated with a $10 \%$ lower risk of CHD death. By assessing the primary incidence of CHD in generally healthy, free-living populations around the world, Del Gobbo et al. [30] also found ALA to be associated with a $9 \%$ lower risk of fatal CHD. From the data extracted from the UK Biobank SOFT CAD GWAS and the CARDIoGRAMplusC4D 1000 Genomes-based GWAS consortia, our results also demonstrate beneficial primary health outcomes for ALA. However, for secondary prevention of CVD, there is little or no effect of ALA, as previously suggested [31], or the evidence is scarce.

Our results, combined with previous findings, support the favorable effects of ALA specifically for the primary prevention of IHD. Mechanistically, these findings are supported by the effects of ALA on improving lipid profile (TC, TG, LDL) [32] and cholesterol homeostasis [33], ameliorating sympathetic heart activity and denervation $[34,35]$, decreasing fasting free fatty acid and inhibiting inflammation and platelet activation [36]. A meta-analysis of 18 observational studies in generally healthy populations found that ALA may be associated with modestly lower risk T2D [37]. Our results also show that genetically-predicted higher plasma ALA is associated with a lower risk of T2D and lower LDL, HDL, TG, and TC. It is

(See figure on next page.)

Fig. 2 Mendelian randomization (MR) analysis testing the effects of n-3 PUFAs on IHD and cardiometabolic risk factors. Results obtained using 4 MR methods (IVW: inverse-variance weighted, WM: weighted median, MR-Egger: Mendelian randomization-Egger, and MR-PRESSO: Mendelian Randomization Pleiotropy Residual Sum and Outlier) are presented as a heat map representing causal estimates (CE). CE of SBP and DBP exceeded that of the others a lot. In order to show the others CE better, CE of SBP and DBP were not included in the heat map. ALA a-linolenic acid, BMI body mass index, DHA docosahexaenoic acid, DPA docosapentaenoic acid, EPA eicosapentaenoic acid, HDL high-density lipoprotein, IHD ischemic heart disease, $L D L$ low-density lipoprotein, MI myocardial infarction, T2D type 2 diabetes, TC total cholesterol, TG triglycerides, WHR waist-to-hip ratio. *Indicates $p<0.05$ for a particular MR approach 


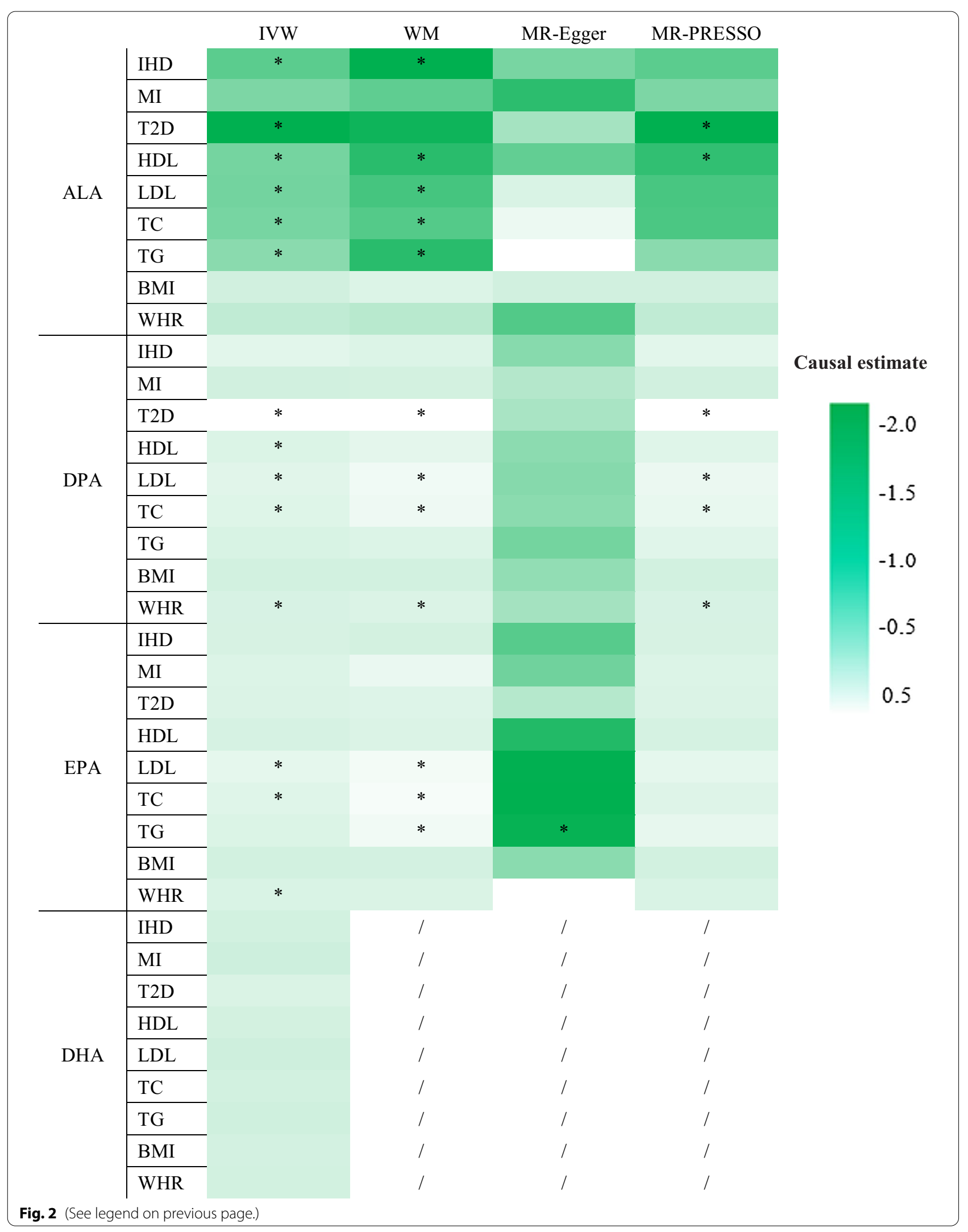


well known that LDL is the initiator of IHD [38], hypertriglyceridemia is the residual risk of IHD $[39,40]$, while diabetes could negatively affect clinical outcomes of IHD, in patients admitted for ST-elevation myocardial infarction (STEMI) [41-43], non-STEMI [44] or stable IHD $[45,46]$. From this perspective, ALA can reduce the risk of IHD in many ways. N-3 PUFAs do not affect atherosclerotic progression, plaque stability, plaque rupture, or thrombosis [10]. This may be related to the ineffectiveness of ALA on MI. Clinically, the phenotypes of MI are not equal to the presence of coronary atherosclerosis. Coronary atherosclerosis may progress as acute coronary thrombotic occlusion or MI, often due to the rupture of an unstable plaque [47-49], usually occurring in plaques with a thin, eroded fibrous cap, regardless of the degree of stenosis $[47,50]$. Many patients live to advanced age with stable, significant IHD and never suffer an MI.

The clinical research on marine-derived n-3 PUFAs (DHA, DPA, and EPA) has been full of twists and turns. Before the use of statins, most of the studies on the cardioprotection of marine-derived n-3 PUFAs were positive [51]. However, after statins became widely used, most studies reported neutral effects [52, 53]. In recent years, the cardioprotective role of marine-derived n-3 PUFAs, especially EPA and DHA, has become increasingly disputed $[54,55]$. Dietary recommendations of EPA and DHA have also been downgraded from Class I to Class II [53], is the reason being that a large number of more recent RCTs $[6,7]$ and integration analyses have found little or no effect of EPA or DHA on cardioprotection $[4,5]$, particularly for primary prevention of CVD $[3,8,9]$. Even when there is an effect, the effect is only seen in studies with a moderate to high risk of bias [56]. Some researchers even think fish oil has disappointing therapeutic benefits [5].

In contrast, other researchers still have hope for marine-derived n-3 PUFAs, especially EPA. They acknowledge that, in over-the-counter formulations (EPA + DHA or fish oil) at common dosages, primary prevention of CVD by marine-derived n-3 PUFAs, is ineffective and that secondary prevention is controversial [10]. They attribute the failure of the previous trials to the low dose and impure formulation of marine-derived n-3 PUFAs, short intervention duration, high background of fish intake, and inappropriate participants [51, 52, 55]. With the large success of the REDUCE-IT trial [57], proponents put their hopes on highly purified EPA (icosapent ethyl), which will lower plasma TG levels, and have given some constructive suggestions for future clinical trials [55]. At this time, the AHA also has given more affirmative recommendations to support the use of marine-derived n-3 PUFAs for reducing the residual risk of CVD that remains after statin therapy [58]. However, as fibrates [59] and PCSK9 inhibitors [60] not only reduce TG, similar to EPA, but also increase HDL and reduce LDL, a new debate arises as to whether we should use fibrates instead of EPA or PCSK9 inhibitors instead of a statin/EPA combination [61]. So, it seems that the debate on the cardioprotection of marine-derived $n-3$ PUFAs will continue.

Our research explores the role of marine-derived n-3 PUFAs from another perspective and finds individual marine-derived n-3 PUFAs have no association with IHD or MI in generally healthy populations. As for CRFs, DPA is associated with a higher risk of T2D and higher HDL, LDL, TC, and WHR; EPA is associated with higher WHR, and DHA does not affect CRFs. Most prior studies showed that, except for reducing TG $[57,61]$, marine-derived n-3 PUFAs do not affect most CRFs or intermediate outcomes $[3,9]$, including high CAD risk factors, i.e. LDL and T2D [9, 37]. Only a few studies have shown that marinederived n-3 PUFAs significantly reduce blood pressure [62], with the greatest reductions in untreated hypertension. Some studies even suggest that marine-derived n-3 PUFAs may increase LDL $[52,58]$, which may negate any cardiovascular benefits [61]. Recently, a large generalpractice RCT show that for patients with multiple cardiovascular risk factors (the criterion was defined as at least four of the following, or for patients with diabetes, at least one of the following: age of 65 years or older, male sex, hypertension, hypercholesterolemia, current smoker, obesity, family history of premature cardiovascular disease), treatment with n-3 PUFAs (1 g DHA + EPA daily, with a median of 5 years of follow-up) did not reduce cardiovascular mortality and morbidity [63].

There are some limitations to our study. First, in our study, both IVs and outcomes come from Europe. This avoids population stratification and conforms to the homogenous principle of an MR study [11]. However, as few n-3 PUFA GWAS are available for African Americans, Chinese, or other races, the potential effects of n-3 PUFAs by race remains to be explored. Similarly, as there are ethnic differences in the risk of cardiovascular disease [64], we need to be cautious in applying our findings to other populations. Second, n-3 PUFAs can be detected in many components of the body (e.g., serum, plasma, phospholipids, cholesterol esters, and adipose tissue) and affect many CVD subtypes (e.g., sudden cardiac death, congestive heart failure, arrhythmia, acute coronary syndrome, and stroke), but our study did not analyze this one by one. A comprehensive analysis of n-3 PUFAs in different components and their association with different disease subtypes may help to reduce potential bias and provide a better understanding of the effect of n-3 PUFAs on cardiovascular health. Third, most instrumental SNPs explain a small proportion variance of n-3 PUFAs. This may 
reduce the power to detect small effects of n-3 PUFAs on IHD risk in our MR framework. Fourth, the effect of n-3 PUFAs on IHD and CRFs found in this study represent a lifelong cumulative effect and are not directly comparable to those derived from conventional observational or clinical studies. Finally, we could not assess whether the effect of n-3 PUFAs on IHD and CRFs varied by sex, age, or the baseline level of n-3 PUFAs as these data are not freely available.

\section{Conclusions}

In summary, this study indicates there are favorable effects of plant-derived ALA on IHD and CRFs, but there is no causal association between marine-derived n-3 PUFAs (DHA, DPA, EPA) and the risk of IHD. Combined with the more affordable, globally accessible, and sustainable plant sources of ALA, compared to marinederived n-3 PUFAs, our study emphasizes the need to further explore the benefits of ALA on IHD. The benefits of marine-derived n-3 PUFAs supplements for cardioprotection remain uncertain and require testing in randomized clinical trials.

\begin{abstract}
Abbreviations
ALA: a-Linolenic acid; BMI: Body mass index; CHD: Coronary heart disease; CRFs: Cardiometabolic risk factors; DBP: Diastolic blood pressure; DHA: Docosahexaenoic acid; DPA: Docosapentaenoic acid; EPA: Eicosapentaenoic acid; GWAS: Genome-wide association studies; HDL: High-density lipoprotein; IHD: Ischemic heart disease; IVs: Instrumental variables; IVW: Inverse variance weighted; LD: Linkage disequilibrium; LDL: Low-density lipoprotein; MI: Myocardial infarction; MR: Mendelian randomization; MR-Egger: Mendelian randomization-Egger; MR-PRESSO: Mendelian Randomization Pleiotropy Residual Sum and Outlier; PUFAs: Polyunsaturated fatty acids; RCT: Randomized controlled trial; SBP: Systolic blood pressure; SNP: Single nucleotide polymorphism; STEMI: ST-elevation myocardial infarction; T2D: Type 2 diabetes; TC: Total cholesterol; TG: Triglycerides; WHR: Waist-to-hip ratio; WM: Weighted Median.
\end{abstract}

\section{Supplementary Information}

The online version contains supplementary material available at https://doi. org/10.1186/s12872-021-02342-6.

Additional file 1: Table S1. Comprehensive results for ALA with $p<5 \times 10^{-8}$. Table S2. Comprehensive results for DHA with $p<5 \times 10^{-8}$. Table S3. Comprehensive results for DPA with $p<5 \times 10^{-8}$. Table S4. Comprehensive results for EPA with $p<5 \times 10^{-8}$. Table S5. Comprehensive results for $n-3$ PUFA with $p<5 \times 10^{-8}$ (removing SNPs in linkage disequilibrium with the other SNPs). Table S6. Association of n-3 PUFA instrumental SNPs with IHD. Table S7. Association of n-3 PUFA instrumental SNPS with MI. Table S8. Association of n-3 PUFA instrumental SNPs with T2D. Table S9. Association of n-3 PUFA instrumental SNPs with LDL. Table S10. Association of n-3 PUFA instrumental SNPS with HDL. Table S11. Association of n-3 PUFA instrumental SNPs with TC. Table S12. Association of n-3 PUFA instrumental SNPs with TG. Table S13. Association of n-3 PUFA instrumental SNPS with SBP. Table S14. Association of n-3 PUFA instrumental SNPs with DBP. Table S15. Association of n-3 PUFA instrumental SNPs with WHR. Table S16. Association of n-3 PUFA instrumental SNPS with BMI. Table S17. Results of MR analyses testing causal effect of n-3 PUFA on IHD and cardiometabolic risk factors.

\section{Acknowledgements}

Data on genetic associations with n-3PUFAs was contributed by the Cohorts for Heart and Aging Research in Genomic Epidemiology (CHARGE) consortium (www.chargeconsortium.com). Data on genetic associations with IHD was from the meta-analysis of UK Biobank SOFT CAD GWAS (interim release) with CARDIoGRAMplusC4D 1000 Genomes-based GWAS (dataset 4) and the Myocardial Infarction Genetics and CARDIoGRAM Exome (dataset 5), contributed by the CARDIoGRAMplusC4D and UK Biobank CardioMetabolic Consortium CHD working group who used the UK Biobank Resource (Nelson CP et al., Nat Genet 2017,49(9): 1385-1391. www.cardiogramplusc4d.org). Data on genetic associations with T2D was contributed by the DIAbetes Genetics Replication and Meta-analysis (DIAGRAM) consortium. Data on genetic associations with lipids was contributed by the Global Lipids Genetics Consortium (GLGC) consortium. Data on genetic associations with blood pressure was contributed by the International Consortium for Blood Pressure (ICBP). Data on genetic associations with BMI and WHR were obtained from the meta-analysis of GWAS for body fat distribution in the UK Biobank and Genetic Investigation of Anthropometric Traits (GIANT). The authors thank all investigators for sharing their data. We also would like to thank Dr. Stanley Li Lin, Department of Cell Biology and Genetics, Shantou University Medical College, for his helpful comments and English language editing.

\section{Authors' contributions}

BX and XT conceived and designed the study. BX acquired all data in the study and was responsible for the integrity of the data. ZX analyzed the data and took responsibility for the accuracy of the statistical analysis. DX interpreted the results. BX and ZX completed the first draft of the manuscript. XT critically revised the manuscript. All authors reviewed the manuscript and approved the final version.

\section{Funding}

This work was supported by Guangdong Medical Research Foundation (No. A2019280), Guangdong Universities Innovation Team Project (Natural, No. 2019KCXTD003), and Li Ka Shing Foundation Cross-Disciplinary Research Grant (No. 2020LKSFG19B).

Availability of data and materials

All datasets generated and/or analyzed during this study are publicly available.

\section{Declarations}

Ethics approval and consent to participate

This study involved analysis of publicly available data and no individual-level data were used. So ethical approval was not required.

\section{Consent for publication}

Not applicable.

\section{Competing interests}

The authors declare that they have no competing interests.

\section{Author details}

${ }^{1}$ Department of Cardiology, First Affiliated Hospital of Shantou University Medical College, Shantou 515041, Guangdong, China. ${ }^{2}$ Department of Medical Service, Second Affiliated Hospital of Shantou University Medical College, Shantou 515041, Guangdong, China. ${ }^{3}$ Clinical Research Center, First Affiliated Hospital of Shantou University Medical College, Shantou 515041, Guangdong, China.

Received: 30 May 2021 Accepted: 22 October 2021

Published online: 08 November 2021

\section{References}

1. Rimm EB, Appel LJ, Chiuve SE, Djousse L, Engler MB, Kris-Etherton PM, et al. Seafood long-chain n-3 polyunsaturated fatty acids and cardiovascular disease: a science advisory from the American Heart Association. Circulation. 2018;138(1):e35-47. 
2. HuY, Hu FB, Manson JE. Marine omega-3 supplementation and cardiovascular disease: an updated meta-analysis of 13 randomized controlled trials involving 127,477 participants. J Am Heart Assoc. 2019;8(19):e013543.

3. Abdelhamid AS, Brown TJ, Brainard JS, Biswas P, Thorpe GC, Moore HJ, et al. Omega-3 fatty acids for the primary and secondary prevention of cardiovascular disease. Cochrane Database Syst Rev. 2020;3:CD003177.

4. Aung T, Halsey J, Kromhout D, Gerstein HC, Marchioli R, Tavazzi L, et al. Associations of omega-3 fatty acid supplement use with cardiovascular disease risks: meta-analysis of 10 trials involving 77917 individuals. JAMA Cardiol. 2018;3(3):225-34.

5. Abbasi J. Another nail in the coffin for fish oil supplements. JAMA. 2018;319(18):1851-2.

6. Kalstad AA, Myhre PL, Laake K, Tveit SH, Schmidt EB, Smith P, et al. Effects of $\mathrm{n}-3$ fatty acid supplements in elderly patients after myocardial infarction: a randomized. Control Trial Circ. 2021;143(6):528-39.

7. Nicholls SJ, Lincoff AM, Garcia M, Bash D, Ballantyne CM, Barter PJ, et al. Effect of high-dose omega-3 fatty acids vs corn oil on major adverse cardiovascular events in patients at high cardiovascular risk: the STRENGTH randomized clinical trial. JAMA. 2020;324(22):2268-80.

8. Manson JE, Cook NR, Lee IM, Christen W, Bassuk SS, Mora S, et al. Marine n-3 fatty acids and prevention of cardiovascular disease and cancer. $N$ Engl J Med. 2019;380(1):23-32.

9. Group ASC, Bowman L, Mafham M, Wallendszus K, Stevens W, Buck G, et al. Effects of n-3 fatty acid supplements in diabetes mellitus. N Engl J Med. 2018;379(16):1540-50.

10. Siscovick DS, Barringer TA, Fretts AM, Wu JH, Lichtenstein AH, Costello RB, et al. Omega-3 polyunsaturated fatty acid (fish oil) supplementation and the prevention of clinical cardiovascular disease: a science advisory from the American Heart Association. Circulation. 2017;135(15):e867-84.

11. Jansen $H$, Samani NJ, Schunkert H. Mendelian randomization studies in coronary artery disease. Eur Heart J. 2014;35(29):1917-24.

12. Lemaitre RN, Tanaka T, Tang W, Manichaikul A, Foy M, Kabagambe EK, et al. Genetic loci associated with plasma phospholipid n-3 fatty acids: a meta-analysis of genome-wide association studies from the CHARGE Consortium. PLoS Genet. 2011;7(7):e1002193.

13. Machiela MJ, Chanock SJ. LDlink: a web-based application for exploring population-specific haplotype structure and linking correlated alleles of possible functional variants. Bioinformatics. 2015;31(21):3555-7.

14. Nelson CP, Goel A, Butterworth AS, Kanoni S, Webb TR, Marouli E, et al. Association analyses based on false discovery rate implicate new loci for coronary artery disease. Nat Genet. 2017:49(9):1385-91.

15. Nikpay M, Goel A, Won HH, Hall LM, Willenborg C, Kanoni S, et al. A comprehensive 1,000 genomes-based genome-wide association metaanalysis of coronary artery disease. Nat Genet. 2015;47(10):1121-30.

16. Webb TR, Erdmann J, Stirrups KE, Stitziel NO, Masca NG, Jansen H, et al. Systematic evaluation of pleiotropy identifies 6 further loci associated with coronary artery disease. J Am Coll Cardiol. 2017;69(7):823-36.

17. Scott RA, Scott $\sqcup$, Magi R, Marullo L, Gaulton KJ, Kaakinen M, et al. An expanded genome-wide association study of type 2 diabetes in europeans. Diabetes. 2017;66(11):2888-902.

18. Willer CJ, Schmidt EM, Sengupta S, Peloso GM, Gustafsson S, Kanoni S, et al. Discovery and refinement of loci associated with lipid levels. Nat Genet. 2013;45(11):1274-83.

19. International Consortium for Blood Pressure Genome-Wide Association S, Ehret GB, Munroe PB, Rice KM, Bochud M, Johnson AD, et al. Genetic variants in novel pathways influence blood pressure and cardiovascular disease risk. Nature. 2011;478(7367):103-9.

20. Pulit SL, Stoneman C, Morris AP, Wood AR, Glastonbury CA, Tyrrell J, et al. Meta-analysis of genome-wide association studies for body fat distribution in 694649 individuals of European ancestry. Hum Mol Genet. 2019;28(1):166-74.

21. Verbanck M, Chen CY, Neale B, Do R. Detection of widespread horizontal pleiotropy in causal relationships inferred from Mendelian randomization between complex traits and diseases. Nat Genet. 2018;50(5):693-8.

22. Bowden J, Davey Smith G, Haycock PC, Burgess S. Consistent estimation in mendelian randomization with some invalid instruments using a weighted median estimator. Genet Epidemiol. 2016;40(4):304-14.

23. Burgess $S$, Thompson SG. Interpreting findings from Mendelian randomization using the MR-Egger method. Eur J Epidemiol. 2017;32(5):377-89.
24. Burgess S, Scott RA, Timpson NJ, Davey Smith G, Thompson SG, Consortium E-I. Using published data in Mendelian randomization: a blueprint for efficient identification of causal risk factors. Eur J Epidemiol. 2015;30(7):543-52.

25. Khankari NK, Murff HJ, Zeng C, Wen W, Eeles RA, Easton DF, et al. Polyunsaturated fatty acids and prostate cancer risk: a Mendelian randomisation analysis from the PRACTICAL consortium. Br J Cancer. 2016;115(5):624-31.

26. Burgess S, Thompson SG, Collaboration CCG. Avoiding bias from weak instruments in Mendelian randomization studies. Int J Epidemiol. 2011;40(3):755-64.

27. Guan W, Steffen BT, Lemaitre RN, Wu JHY, Tanaka T, Manichaikul A, et al. Genome-wide association study of plasma N6 polyunsaturated fatty acids within the cohorts for heart and aging research in genomic epidemiology consortium. Circ Cardiovasc Genet. 2014;7(3):321-31.

28. Stock JH, Wright JH, Yogo M. A survey of weak instruments and weak identification in generalized method of moments. J Bus Econ Stat. 2002;20(4):518-29.

29. Pan A, Chen M, Chowdhury R, Wu JH, Sun Q, Campos H, et al. AlphaLinolenic acid and risk of cardiovascular disease: a systematic review and meta-analysis. Am J Clin Nutr. 2012;96(6):1262-73.

30. Del Gobbo LC, Imamura F, Aslibekyan S, Marklund M, Virtanen JK, Wennberg M, et al. Omega-3 polyunsaturated fatty acid biomarkers and coronary heart disease: pooling project of 19 cohort studies. JAMA Intern Med. 2016;176(8):1155-66.

31. Abdelhamid AS, Brown TJ, Brainard JS, Biswas P, Thorpe GC, Moore HJ, et al. Omega-3 fatty acids for the primary and secondary prevention of cardiovascular disease. Cochrane Database Syst Rev. 2018;11:CD003177.

32. Deng Q, Yu X, Xu J, Kou X, Zheng M, Huang F, et al. Single frequency intake of alpha-linolenic acid rich phytosterol esters attenuates atherosclerosis risk factors in hamsters fed a high fat diet. Lipids Health Dis. 2016;15:23.

33. O'Reilly ME, Lenighan YM, Dillon E, Kajani S, Curley S, Bruen R, et al. Conjugated linoleic acid and alpha linolenic acid improve cholesterol homeostasis in obesity by modulating distinct hepatic protein pathways. Mol Nutr Food Res. 2020;64:e1900599.

34. Sardu C, Santulli G, Santamaria M, Barbieri M, Sacra C, Paolisso P, et al. Effects of alpha lipoic acid on multiple cytokines and biomarkers and recurrence of atrial fibrillation within 1 year of catheter ablation. Am J Cardiol. 2017;119(9):1382-6.

35. Marfella R, Barbieri M, Sardu C, Rizzo MR, Siniscalchi M, Paolisso P, et al. Effects of alpha-lipoic acid therapy on sympathetic heart innervation in patients with previous experience of transient takotsubo cardiomyopathy. J Cardiol. 2016;67(2):153-61.

36. Joris PJ, Draijer R, Fuchs D, Mensink RP. Effect of alpha-linolenic acid on vascular function and metabolic risk markers during the fasting and postprandial phase: A randomized placebo-controlled trial in untreated (pre-)hypertensive individuals. Clin Nutr. 2019.

37. Wu JH, Micha R, Imamura F, Pan A, Biggs ML, Ajaz O, et al. Omega-3 fatty acids and incident type 2 diabetes: a systematic review and meta-analysis. Br J Nutr. 2012;107(Suppl 2):S214-27.

38. Akhmedov A, Sawamura T, Chen CH, Kraler S, Vdovenko D, Luscher TF. Lectin-like oxidized low-density lipoprotein receptor-1 (LOX-1): a crucial driver of atherosclerotic cardiovascular disease. Eur Heart J. 2021;42(18):1797-807.

39. Vallejo-Vaz AJ, Corral P, Schreier L, Ray KK. Triglycerides and residual risk. Curr Opin Endocrinol Diabetes Obes. 2020;27(2):95-103.

40. Ji Y, Bai C. Research progress of hypertriglyceridemia and coronary heart disease. Heart Mind. 2018;2:40-4.

41. Marfella R, Rizzo MR, Siniscalchi M, Paolisso P, Barbieri M, Sardu C, et al. Peri-procedural tight glycemic control during early percutaneous coronary intervention up-regulates endothelial progenitor cell level and differentiation during acute ST-elevation myocardial infarction: effects on myocardial salvage. Int J Cardiol. 2013;168(4):3954-62.

42. Marfella R, Sardu C, Balestrieri ML, Siniscalchi M, Minicucci F, Signoriello G, et al. Effects of incretin treatment on cardiovascular outcomes in diabetic STEMI-patients with culprit obstructive and multivessel non obstructivecoronary-stenosis. Diabetol Metab Syndr. 2018;10:1.

43. Sardu C, Barbieri M, Balestrieri ML, Siniscalchi M, Paolisso P, Calabro P, et al. Thrombus aspiration in hyperglycemic ST-elevation myocardial infarction 
(STEMI) patients: clinical outcomes at 1-year follow-up. Cardiovasc Diabetol. 2018;17(1):152.

44. Marfella R, Sardu C, Calabro P, Siniscalchi M, Minicucci F, Signoriello G, et al. Non-ST-elevation myocardial infarction outcomes in patients with type 2 diabetes with non-obstructive coronary artery stenosis: effects of incretin treatment. Diabetes Obes Metab. 2018;20(3):723-9.

45. Sasso FC, Pafundi PC, Marfella R, Calabro P, Piscione F, Furbatto F, et al. Adiponectin and insulin resistance are related to restenosis and overall new $\mathrm{PCl}$ in subjects with normal glucose tolerance: the prospective AIRE Study. Cardiovasc Diabetol. 2019;18(1):24.

46. Sardu C, Paolisso P, Sacra C, Mauro C, Minicucci F, Portoghese M, et al. Effects of metformin therapy on coronary endothelial dysfunction in patients with prediabetes with stable angina and nonobstructive coronary artery stenosis: the CODYCE multicenter prospective study. Diabetes Care. 2019:42(10):1946-55.

47. Galis ZS, Sukhova GK, Lark MW, Libby P. Increased expression of matrix metalloproteinases and matrix degrading activity in vulnerable regions of human atherosclerotic plaques. J Clin Invest. 1994;94(6):2493-503.

48. Libby P. What have we learned about the biology of atherosclerosis? The role of inflammation. Am J Cardiol. 2001:88(7B):3J-6J.

49. Libby P. Coronary artery injury and the biology of atherosclerosis: inflammation, thrombosis, and stabilization. Am J Cardiol. 2000;86(8B):3J-8J (discussion J-9J)

50. van der Wal $A C$, Becker $A E$, van der Loos CM, Das PK. Site of intimal rupture or erosion of thrombosed coronary atherosclerotic plaques is characterized by an inflammatory process irrespective of the dominant plaque morphology. Circulation. 1994;89(1):36-44.

51. Kris-Etherton PM, Richter CK, Bowen KJ, Skulas-Ray AC, Jackson KH, Petersen KS, et al. Recent clinical trials shed new light on the cardiovascular benefits of omega-3 fatty acids. Methodist Debakey Cardiovasc J. 2019:15(3):171-8

52. Hilleman DE, Wiggins BS, Bottorff MB. Critical differences between dietary supplement and prescription omega-3 fatty acids: a narrative review. Adv Ther. 2020;37(2):656-70.

53. Elagizi A, Lavie CJ, Marshall K, DiNicolantonio JJ, O'Keefe JH, Milani RV. Omega-3 polyunsaturated fatty acids and cardiovascular health: a comprehensive review. Prog Cardiovasc Dis. 2018;61 (1):76-85.

54. Maki KC, Dicklin MR. Omega-3 fatty acid supplementation and cardiovascular disease risk: glass half full or time to nail the coffin shut? Nutrients. 2018;10(7):864.
55. Jia X, Kohli P, Virani SS. Omega-3 fatty acid and cardiovascular outcomes: insights from recent clinical trials. Curr Atheroscler Rep. 2019;21(1):1.

56. Hooper LAA, Ajabnoor S, Brainard J, Brown T, Hanson S, et al. Set of systematic reviews of RCTs on the health effects of omega 3 polyunsaturated fats in adults. Geneva: World Health Organization; 2017.

57. Bhatt DL, Steg PG, Miller M, Brinton EA, Jacobson TA, Ketchum SB, et al. Cardiovascular risk reduction with icosapent ethyl for hypertriglyceridemia. N Engl J Med. 2019;380(1):11-22.

58. Skulas-Ray AC, Wilson PWF, Harris WS, Brinton EA, Kris-Etherton PM, Richter CK, et al. Omega-3 fatty acids for the management of hypertriglyceridemia: a science advisory from the American Heart Association. Circulation. 2019;140(12):e673-91.

59. Jakob T, Nordmann AJ, Schandelmaier S, Ferreira-Gonzalez I, Briel M. Fibrates for primary prevention of cardiovascular disease events. Cochrane Database Syst Rev. 2016;11:CD009753.

60. Schwartz GG, Steg PG, Szarek M, Bhatt DL, Bittner VA, Diaz R, et al. Alirocumab and cardiovascular outcomes after acute coronary syndrome. N Engl J Med. 2018;379(22):2097-107.

61. Doggrell SA. Clinical trials of eicosapentaenoic acid (EPA) prescription products for the treatment of hypertriglyceridemia. Expert Opin Pharmacother. 2019;20(10):1221-5.

62. Miller PE, Van Elswyk M, Alexander DD. Long-chain omega-3 fatty acids eicosapentaenoic acid and docosahexaenoic acid and blood pressure: a meta-analysis of randomized controlled trials. Am J Hypertens. 2014;27(7):885-96.

63. Risk, Prevention Study Collaborative G, Roncaglioni MC, Tombesi M, Avanzini F, Barlera S, et al. n-3 fatty acids in patients with multiple cardiovascular risk factors. N Engl J Med. 2013;368(19):1800-8.

64. Conklin Al, Tam AC, Yao CA, Guo SX, Richardson CG. Ethnic inequalities in cardiovascular disease risk: strength of ethnic identity predicts obesity prevalence in late adolescence. Heart Mind. 2018;2:98-105.

\section{Publisher's Note}

Springer Nature remains neutral with regard to jurisdictional claims in published maps and institutional affiliations.

Ready to submit your research? Choose BMC and benefit from

- fast, convenient online submission

- thorough peer review by experienced researchers in your field

- rapid publication on acceptance

- support for research data, including large and complex data types

- gold Open Access which fosters wider collaboration and increased citations

- maximum visibility for your research: over $100 \mathrm{M}$ website views per year

At BMC, research is always in progress.

Learn more biomedcentral.com/submissions 